\title{
Peri-Implantitis In Dental Implants: An Updated Review
}

\author{
Duggal N¹, Bhayana $\mathbf{G}^{2}$, Juneja $\mathrm{A}^{3}$, Puri $\mathbf{M}^{4}$, Kumar $\mathrm{A}^{5}$, Dahiya $\mathrm{A}^{6}$, SharmaV7
}

\begin{abstract}
The use of dental implants has revolutionized the treatment of partially and fully edentulous patients today. While in many cases dental implants have been reported to achieve long-term success, these are also not immune from the complicationsincludingperi-implant mucositis and peri-implantitis. Peri-implantitis is an inflammatory process which involves bone loss around osseointegrated implant in function. The etiology of the disease has been attributed to bacterial infections, occlusal overload, surgical trauma, faulty or incorrect prosthetic design and/ or improper implant placement. Diagnosis is based on changes in colour of the gingiva, bleeding and probing depth of peri-implant pockets, suppuration, $x$-ray and gradual loss of bone height around the tooth. Treatment modalities will differ depending upon whether it is a case of periimplantmucositis or periimplantitis, Therapeutic objectives focus on control of infection, detoxification of the implant surface and regeneration of the alveolar bone. This review article gives a brief description of etiopathogenesis, diagnosis and various treatment options in the management of periimplant disease.
\end{abstract}

Key Words : Dental implant, Peri-implantitis, Peri-implant mucositis.

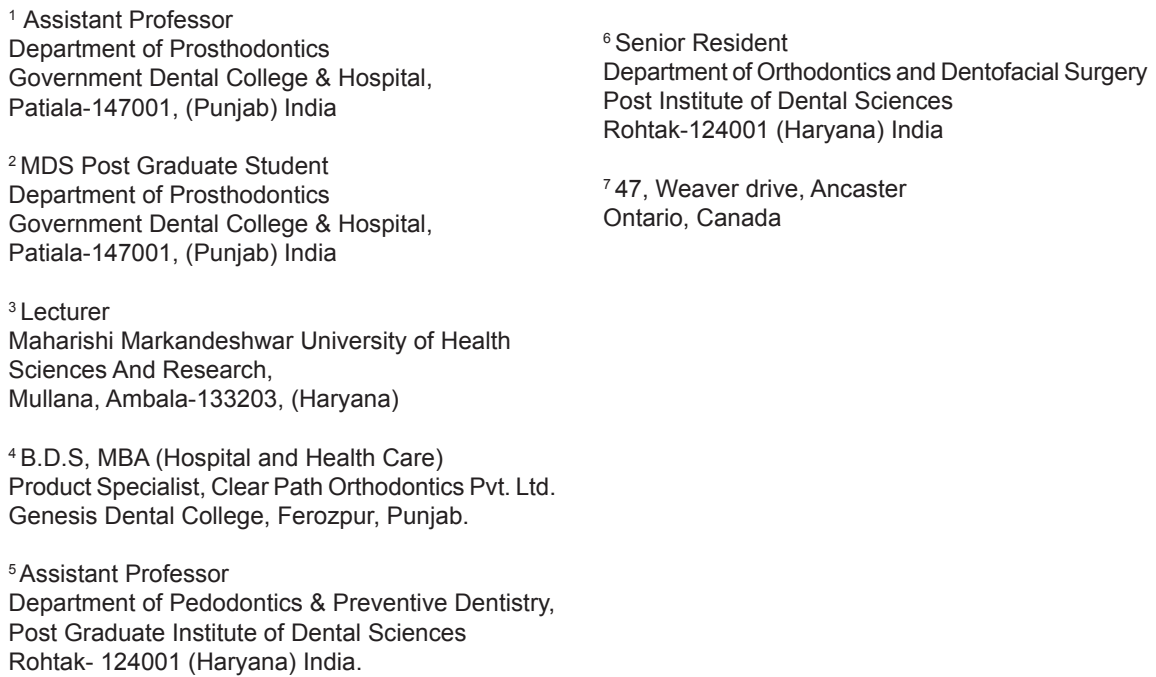

${ }^{6}$ Senior Resident

Department of Orthodontics and Dentofacial Surgery

Post Institute of Dental Sciences

${ }^{7} 47$, Weaver drive, Ancaster Ontario, Canada

\section{Contact Author}

\section{Dr. Arun Kumar}

drarun922@gmail.com

\section{INTRODUCTION}

7 ehabilitation of partially and totally edentulous patient with 1 dental implants has become common. Apart from this, dental implants have become treatment approach for managing a broad range of clinical dilemmas due to their highly predictable results and their ability to be used for a wide variety of treatment options. Inspite of their success, it has been documented that over a 5 years period of time, 0 to $14.4 \%$ of dental implants demonstrated peri-implant inflammatory reactions which were associated with crestal bone loss that may eventually lead to the loss of an implant (1).

Peri-implant disease refers to the pathological inflammatory changes that take place in the tissues surrounding a load bearingimplant (2). Peri-implant disease presents in two formvizperiimplant mucositis and peri-implantitis. 
Peri-implant mucositis is a disease in which the presence of inflammation is confined to the soft tissues surrounding a dental implant with no signs of loss of supporting bone following initial bone remodeling during healing.Peri-implantitis is defined as an inflammatory process which affects the tissues around an osseointegrated implant in function, resulting in the loss of supporting bone, which is often associated with bleeding, suppuration, increased probing depth, mobility and radiographical bone loss.

\section{ETIOPATHOGENESIS}

- Periimplant Tissue MorphologyEpithelial fibers surrounding dental implants are arranged predominantly longitudinal to the implant surface. In addition to this, low degree of vascularization and a higher collagen fiber to fibroblast ratio in comparison to natural tooth (a ratio of 4 in natural tooth and 109 in the implant) is present. If this weak permucosal seal is destroyed, bacterial contamination spreads directly to the bone, leading to its rapid destruction.

- Implant structure-The design of implant play an important role in the onset of peri-implantitis. As Binon et al. described in their study, there is a difference of between 20 and 49 micro between the components of different types of implants currently available in the market (3). This space provides enough room for the entry of micro-organisms of oral flora measuring less than 10 micro. Thus poor alignment of the components of implant may enhance retention of microbial plaque.Another reported cause of peri-implantitis is the corrosion that can occur when a non-noble metal structure is connected to a titanium implant. This causes increased rush of macrophages in peri-implant tissues, which would favour the initial bone resorption due to non-infectious cause.

- Microbial infection- Bacterial flora at failing implants are gram negative anaerobes like Prevotella intermedia, Porphyromonasgingivalis, Actinobacillusactinomycetamcomitans, Bacteroidesforsythus, Treponemadenticola, Prevotellanigrescenes, Peptostreptococcus micros and Fusobacterium nucleatum $(4,5)$ which are similar to those found around natural teeth in patients with severe chronic periodontitis.Implants in partially edentulous patients with active periodontal disease are at greater risk of peri-implantitis than that of completely edentulous patients. This is possible because gingival sulcus of natural tooth serve as reservoir for periodontal pathogens which maylater colonizes the implant present in the same mouth.

\section{BIOMECHANICAL FACTORS}

Excessive mechanical stresses play a significant role in the failure of an implant. Implants are not able to tolerate axial or lateral stresses as well as natural teeth owing to theresiliency of only half of a tooth (100 micro in natural tooth, 50 micro for implant) (6). Microfractures appear in the bone around an osseointegrated implant when subjected to excessive load. On occasions, these forces cause a prosthetic component (resin, ceramic or the transepithelial abutment screw) or the implant itself to fracture.

Excessive mechanical stresses can be the consequence of occlusal over load, poor treatment regime or improper relationship between crown length and implant length.Other risk factors include previous periodontal disease, poor plaque control / inability to clean, residual cement, smoking, genetic factors, drug abuse, parafunctional habits, radiation, chemotherapy, long term corticosteroid therapy, systemic diseases e.g. diabetes mellitus, osteoporosis, rheumatic arthritis with concomitant connective tissue disease, iatrogenic factors such as lack of primary stability and premature loading.

\section{DIAGNOSIS}

There is no single diagnostic tool that can, with certainty, establish a diagnosis of peri-implantitis. Hence different diagnostic parametric should be used and correlated for early detectionof peri-implantitis.

\section{Probing, Bleeding and Suppu- ratio}

Probing of implant should be done initially during the placement of implant using light force of $0.25 \mathrm{~N}$ with a blunt straight plastic probe such as automated probe or TPS probe. Probing depth should be recorded for future referral. Gentle probing resulting in bleeding suggests the presence of soft tissue inflammation. Increasing probing depth or suppuration are indicators of pathological changes and necessitate further evaluation and treatment.

\section{Radiograph}

Periapical intraoral radiograph of implant following placement of implant and following prosthesis installation should function as the baseline to which all the future radiographs are to be compared. Progressive bone loss is a definitiveindicator of peri-implantitis. However, on X-ray, problem can be detected only when $30 \%$ of bone mass has been lost and also,improperly placed X-ray can lead to errors in determining the size and morphology of bony defects. Thus, radiograph should be placed perpendicular to implant body to avoid any distortion or other radiographic techniques such as CBCT must be considered for more accurate determination of defect.

\section{Mobility}

It should not be considered as a good diagnostic aid since prognosis of mobile implant is hopeless and should be removed. However, interpretation of low degree mobile implant can be done with an electronic device like periotest. Perceived implant mobility can also be related to loosened restoration and/ or abutment component, which may contribute to plaque accumulation and further development of peri-implant 
mucosistis and / or peri-implantitis.

\section{Other diagnostics}

Bacterial culture, inflammatory markers, DNA probes, polymerase chain reaction (PCR), monoclonal antibody, enzyme assays, recording gingival temperature and peri-implant fluid volume are other useful diagnostic tools for the diagnosis of peri-implant disease.

\section{MANAGEMENT}

With the continuously increasing number of implants placed in everyday clinical practice, it is reasonable to anticipate an increasing prevalence of peri-implantitis, which underlines the necessity for a predictable therapy. The treatment protocol will differ depending on whether it is mucositis or peri-implantitis.

If there is no bone loss i.e. in case of mucositis, local plaque removal is done with plastic instruments (Implacar) followed by polishing with rubber cup and paste, floss, inter-dental brushes. Chemical plaque control can be achieved with $0.12 \%$ chlorhexidine applied topically, every 8-12 hours for 15 days. Patient should also be instructed about optimum oral hygiene maintenance. Prosthesis design should also be checked and corrected, in order to remove any design defect impeding proper oral hygiene or producing increased biomechanical stress. Once this initial phase of treatment is completed patient should be regularly recalled for periodic check-ups.

If peri-implantitis is diagnosed, treatment will largely depend on esthetic requirements of the implant and amount of bone loss. If bone loss is in initial stages, treatment protocol will be similar to that of mucositis, with addition of decontamination of implant surface and antibiotics. If bone loss is advanced or persists despite initial treatment, it will be necessary to surgically debride the soft peri-implant tissues, decontaminate implant surface and finally apply regenerative procedures using a bone graft substitute in combination with a method to remove the lost bone.

\section{Implant surface decontamina- tion}

Photodynamic therapy is a noninvasive method that could be used to reduce microorganisms in peri-implantitis. Conventional hand and ultrasonic instruments are not suitable for preparation and detoxification of the implant surface Prophyjet, the use of high pressure air powder abrasive (mixture of sodium bicarbonate particles with sterile water) is the best way to eliminate endotoxins and remains from all surfaces. Other chemotherapeutic agents like saturated solution of citric acid ( $40 \%$ concentration, ph 1$)$ for $30-60$ seconds is an effective means of decontamination for hydroxyapatite coated implants, where chlorhexidine is not much effective. Machined titanium surface can be decontaminated with the use of topical tetracycline (one $250 \mathrm{mg}$ capsule mixed with saline serum until a creamy consistency is obtained). Tetracycline stimulates fibroblast growth in the affect area.

Prolonged application of citric acid solution on hydroxyapatite surfaces is not recommended, since this would impair its ability to bond to the titanium body of the implant.

If hydroxyapatite surface is already damaged due to infection around the implant, it is recommended to completely eliminate it by drilling and then proceed to apply air abrasion or ultrasound followed by decontamination with tetracycline in the same manner as it were a machined titanium surface. Soft laser irradiation can also be used as a method of decontamination for different implant surfaces. The semiconductor $809 \mathrm{~nm}$, the CO2 and erbium-doped:yttrium, aluminum and garnet (ER: YAG) lasers are recommended.

\section{Anti-infective therapy}

Treatment of peri-implant infection by mechanical debridement with plastic curettes combined with antibiotic therapy ( $0.2 \%$ chlorhexidine) may lead to improvement in deep peri-implant lesions with mean pocket probing depth more than $5 \mathrm{~mm}$. Additionally, patients suffering from localized periimplant problems are the candidates for local-drug delivery devices, Actisitie (fiber containing polymeric tetracycline $\mathrm{HCl}$ ) has shown significantly lower total anaerobic counts.

If the problem is generalized, specific microbiological information must be collected and antibiotic are administered systemically. The recommended oral antibiotic treatment consists of: amoxicillin, amoxicillin plus clavulanic acid, amoxicillin associated with metronidazole or, in the case of penicillin - allergic patients, erythromycin and tetracycline. The standard treatment time is 7 and 10 days (7).

\section{Surgical Technique}

Surgical treatment will depend on the amount of bone lost and aesthetics. Treatment for unaesthetic zone, horizontal bone loss and moderate bony defects (smaller than $3 \mathrm{~mm}$ ) is ginivectomy and apically displaced flaps, in order to reduce pocket depth and improve access for oral hygiene.

If the vertical $(<3 \mathrm{~mm})$ one to two wall defects are present, then respective surgery can be done to reduce the pockets, to smoothen the rough implant surface, to correct the bony architecture and to increase the area of the keratinized gingival (8). To arrest the progression of disease and to aid in maintenance of oral hygiene smoothing and polishing rough surfaces or elimination of implant thread is done. This technique is known as implantoplasty.In case of three wall circumferential defects or when the implant is strategicallyimportant in conserving the prosthesis or, if it is present in aesthetic zone, guided bone regeneration techniques or other various bone graft technique, even in conjugation with 
platelet richplasma (PRP) can be used. Prosthesis is removed and thorough decontamination of implant surface is done followed by elaborate irrigation with saline solution. Roughening of implant surface can also be done with round burs to increase the accessibility of the osteogenic cells and membranes are placed undisturbed for 4-6 weeks. Intra-oral autogenous bone grafts are the most preferred type of grafts for guided bone regeneration therapies (9). Other bone grafts, like demineralized freeze-dried bone and hydroxyapatite can also be used.

\section{CONCLUSION}

Peri-implantitis is a site specific infectious disease that causes an inflammatory process in soft tissues and bone loss around an osseointegrated implant in function. Etiology of peri-implant disease is conditioned by the status of the tissue surrounding the implant, implant design, location, diameter and number of implants, degree of roughness, excessive mechanical load, faulty or incorrect prosthetic design and / or improper surgical placement. Gram negative anaerobes play a major role in this disease. Several treatment modalities are advocated, but however, there is still insufficient evidence to support an ideal universal therapy for peri-implanttits. Therefore, it appears reasonable to attempt the interception of destructive peri-implantitis as early as possible.

\section{REFERENCES}

1. Bergbundh T, et al. A Systematic review of the incidence of biological and technical complications in implant dentistry. $J$ ClinPeriodontol 2002;29:197-212.

2. Mombelli A, Lang NP. The diagnosis and treatment of peri-implantitis. Periodontol 2000;1998;17:63-76.

3. Binon P, Weir D, Watanabe L, Walker L. Implant component compatibility. En: Laney WR, Tolman DE (eds). Tissue integration in oral orthopedic and maxillofacial reconstruction. Chicago: Quintessence 1992; p. 218-226.

4. Heydenrijk K, Meijer HJA, Van der Reijden WA, Raghoebar GM, Vissink A, Stegenga B. Microbiota around root-form endosseous implants: a review of the literature. Int J Oral Maxillofac Implants 2002,17:829-38.

5. Shibli JA, Compagnoni Martins M, Moreira Lotufo RF, Marcantonio E, Microbiologic and radiographic análisis of ligature-induced periimplantitis with different dental implant surfaces, Int J Oral Maxillofac Implants 2003;18:383-90.

6. Bobia F, Pop RV. Periimplantitis. Aetiology, diagnosis, treatment. A review from the literature, Current Health Sciences Journal 2010,36:3;171-75.

7. Zablotsky $\mathrm{NH}$, Diedrich DL, Meffert RM. Detoxificacion of endotoxincontaminatedtitanium and hydroxyapatite-coated surfaces utilizing various chemotherapeutic and mechanical modalities. Implant Dent 1992;1:154-158

8. Jovanoic SA. The management of periimplant breakdown around functioning osseointegrated dental implants. $J$ Periodontol 1993;64:1176

9. Newman HG, Takei HH, Carranza. Clinical Periodontology, 9th Edition,1987 p. 936940. 